\title{
Reproductive System Findings Sponsor Defined Identifier
}

National Cancer Institute

\section{Source}

National Cancer Institute. Reproductive System Findings Sponsor Defined Identifier. NCI

Thesaurus. Code C117639.

One or more sponsor defined characters used to identify, name, or characterize the reproductive system finding. 